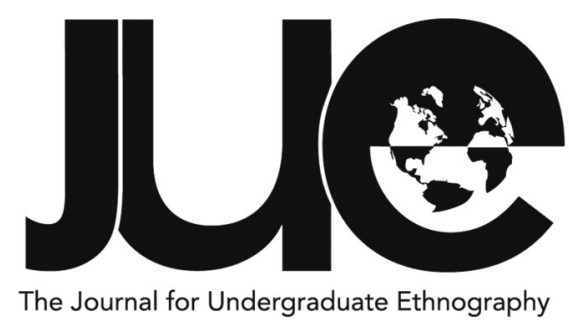

\title{
Medicalization and Fear: A Midwifery View of the Phenomenon and the Backlash
}

\author{
Sydney Comstock
}

Wake Forest University, sydneycomstock@alumni.wfu.edu

\section{ABSTRACT}

The phenomenon of medicalization in the United States is something that midwives must deal with on a daily basis, and it has far-reaching consequences for women's health. This article examines the culture of birth in the U.S. and how medicalization has manifested itself as a social norm from the perspectives of working certified nurse midwives in hospitals and birth centers. It explores the philosophy of the medicalized birth, the impact of technology on the perpetuation of medicalization in United States' culture, and the fear of this phenomenon that midwives are starting to see in practice, which adversely affects their work. This article argues that advances in and dependence on obstetrical technology have enabled medicalization to continue and created a response of fear from women who worry this phenomenon will negatively affect their birthing experience. My research demonstrates that midwives recognize that the dominance of technology in health care has shaped not only how birth has become medicalized, but also how women are responding to this "technocratic birth" and how navigating women's fears about hypermedicalization has become a central part of midwives' practice. Through Michel Foucault's theory of biopower and Robbie Davis-Floyd's idea of the "technocratic birth," this article explains how medicalization depends on technology and why midwives are seeing an adverse reaction from women who fear these trends.

Keywords: birth; medicalization; midwifery; women's health; United States 
Y ou know things are done to people, instead of decisions being made with people, like a hallmark of midwifery care is being with women and not doing things to them...like, that's an important part of midwifery education. I think that's like most midwives would really talk about how much of their practice is around helping women feel heard and understood and participate in decision making processes and the owners of their own experience and care, and those things. And that it's not that you would never hear a physician talk about those things, and I think that's very unfair to suggest that ob-gyns don't, but it's not part of their practice to the same extent. I mean, one way of sort of talking about it and thinking about it is that, like, for a physician everything is wrong with the pregnancy until proven right and the only way to be proven right is that everything is okay is to have a baby, so for midwives everything is healthy and normal until proven otherwise. (Midwife Interview 2)

In the past twenty years, the demand for midwives to attend births has steadily increased. From the year 1989 to 2018, the number of births attended by certified nurse midwives in the U.S. rose from 3.3\% to $9.0 \%$ (Centers for Disease Control and Prevention 2020; Declercq 2015). The midwifery model, which incorporates the patient-centered healthcare model, and the biomedical model of pregnancy or the focus on the body's physical processes, characterized by hypermedicalization, are seen as competing models within the U.S. system. "Medicalized birth" is the phenomenon where medical interventions are viewed as routine during childbirth in Western countries, specifically the United States, without evidence of constant effectiveness. This is illustrated by the high rate of cesarean sections, high rates of inductions, and reliance on technological interventions during the birthing experience Johanson, Newburn, and Macfarlane 2002). Due to women's increased desire for control and decision-making power over their birth process, midwives see the opposite side of medicalization with women going against medical advice for fear of falling victim to a medicalized birth with unnecessary interventions. I argue that medicalization continues in the United States because of the dependence that birthing techniques have on technology, which in turn has created a unique form of resistance as women push back against the biomedical model during their birth experience.

Midwives are typically older women who have already had their own children, located in all parts of the world to assist women in their community going through the childbirth process (Connerton 2012). As technology has changed throughout time, specifically with the invention of forceps, men began to enter the field of birth and eventually created the field of obstetrics, which was born in Europe and then made its way to the United States (Brodsky 2008). Since men were the only ones who could go to medical school and use these technologies in the 1800s, this signaled a shift away from the traditional birth model of a midwife attendant helping women give birth at home toward birthing with surgeons in hospitals who used the newest technology of the day (Capitulo 1998). Surgeons focused their efforts on gaining upper-class women as clientele for hospital births, knowing these individuals could pay for their services and left midwives to continue providing care for less fortunate families (Capitulo 1998). This focus on specific consumers created a connotation of midwives as being for the poorer cohorts of society while male obstetric providers served the rich, which these physicians then exploited to increase their already high status in society (Capitulo 1998).

Male surgeons began to insist that it was safer and healthier for women to have their babies in the hospitals because they had access to pain relief and new technologies. They eventually went so far as to demonize mothers who decided against this model, pushing 
midwives further from hospitals (Connerton 2012). However, midwives never fully disappeared due to their use by rural and lowincome women. This led the Frontier School for Nursing, an early school for nurses and midwives that focused on rural populations, to incorporate midwives into their nursing programs, which kept the field going throughout the middle of the twentieth century (Connerton 2012). During the 1970s and 1980s, there was another shift as women began wanting to have their children in birth centers and to be attended by midwives in response to the hyper-medicalization happening in hospitals reliant on technologies (Capitulo 1998). Women and midwives began criticizing certain aspects of the hyper-medicalization of birth, including aggressive technological dominance over the birthing process that left women with no control over the process their bodies go through. Today, women of higher socioeconomic status have more agency to seek out and to pay for midwives as attendants to their births, but this has not changed the overall trend of women in the United States deciding to give birth in hospitals with a doctor (Martin et al. 2019).

According to federal statistics of birth and death data in the United States, the percentage of the total babies delivered by certified nurse midwives is $9.1 \%$, with the rest of the births assisted by physicians (Centers for Disease Control and Prevention 2020; Martin et al. 2019). In 2017, the percentage of women who gave birth outside of a hospital, whether that be at home or in a birth center, is $1.61 \%$ (MacDorman and Declercq 2019). According to the CDC (2019), in 2018, the percentage of all births in the United States that were done by Csection was $31.9 \%$. The World Health Organization (2015) made it clear in 1985 and then reaffirmed in 2015 that countries should aim for a C-section rate from $10-15 \%$, as anything higher does not improve outcomes for mothers. These statistics of procedures happening in hospitals that depend on the newest technologies indicate the medicalization that continues and why women are fearful of this affecting their birth. This picture of birth in the United States illustrates the rise of medicalization due to a dependence on technology. Moreover, this process of medicalization will demonstrate why midwives see women, at times dangerously, reject a biomedical interference in their birthing experience.

\section{Definitions}

Medicalization is the change of a normal process into a pathology, bringing an experience into the realm of western medicine and subjecting it to the authority of medical professionals (Parry 2008). One midwife explains how she "think[s] we're so quick to like test 60 things [when] we really could just be testing one thing, and we're not like... we're over testing, we're over monitoring, or over inducing. That's, that's what medicalization means to $\mathrm{me}^{\prime \prime}$ (Midwife Interview 8). In the United States, birth is a cultural phenomenon wherein people treat the childbearing process as a move away from a normal bodily state, one which requires constant medical care under the purview of the medical establishment because it is considered dangerous. Once a natural process goes through medicalization, it then becomes subject to the authority of medical institutions and professionals, whom we look toward for answers about our conditions (Parry 2008). Birth becomes medicalized through the fear and risk associated with it in U.S. culture. These events are then described in medical terminology that only doctors understand; people are seen less as individuals in a cultural context and instead viewed as patients through a medical gaze (Parry 2008). For example, midwives support birth starting spontaneously and on its own, without medical intervention; however, we have the technology to induce birth and many providers use this if the patient requests the procedure. Additionally, the constant use of fetal heart monitors during birth rather than using them to check in intermittently as midwives typically do is a form of medicalization. All of these actions create a conceptualization of the female body as a machine and remove a woman from the happenings of her experience (R. Davis-Floyd 1987). These differences in parts of the birth process affect midwives navigating the world of medicalization in the United States.

Midwifery is not always well known within the United States as there are multiple different 
types within the profession, and they differ in what type of training they go through. A certified nurse midwife (CNM) is an individual who has gone to a graduate-level midwifery program that is accredited by the Accreditation Commission for Midwifery Education (ACME) (American College of Midwives n.d.). Every CNM must pass a national examination that is administered by the American Midwifery Certification Board (AMCB) to be able to practice in all 50 states (American College of Midwives n.d.). A certified professional midwife (CPM), or lay midwife, has gone through more of an apprenticeship and is certified by the North American Registry of Midwives (American College of Midwives n.d.). Each state has different laws and rules about how CPMs can practice. This research deals mainly with CNMs, but it is important to differentiate within the profession itself. A birth center is a space that is outside of the hospital where women can pay to deliver their babies and is typically run by midwives and other birth attendants, such as doulas and nurses. A birth attendant is a very general term for any health professional who gives care to a mother and newborn during and immediately following a birth. These services are typically paid for by insurance if the plan covers a midwife, or patients pay out of pocket.

\section{Methods}

I conducted seven semi-structured interviews with currently practicing certified nurse midwives. I located the individuals through existing networks with midwives and doulas, which I then continued through snowball sampling. These networks were established through previous research and professional connections. I reached out to them through email, asking them to read and sign a consent form, which was approved by Wake Forest University's Institutional Review Board. Four of the seven have been practicing for at least five years, and three have been practicing midwives for shorter but have been doulas and birth attendants for years prior. All of the participants were women and ranged in age and experience from more recently graduated to practicing for twenty years. Each interview lasted 45 to 75 minutes. All interviews were conducted in person and took place in coffee shops, interviewees' homes, or offices.
The interviews were transcribed and then coded by highlighting quotations and sorting them into different patterns identifying medicalization or the backlash from it. There were a set of questions that worked as a starting point to guide the semi-structured interviews and aimed to get answers pertaining to real-life experiences. These questions encompassed topics such as personal background, professional experience, medicalization issues, and different situations in their daily work. Although this interview experience was daunting at first, these midwives care immensely about their work and were eager to answer my questions, many times answering them during our conversation without me having to ask.

\section{Medicalized Birth and Fear}

Medicalization in the United States today has been driven by new technologies that aim to make birth less dangerous and unpredictable, women requesting these new innovations, and physicians suggesting them at higher rates. However, midwives see two sides to this cultural phenomenon. The first side is the typical embodiment of medicalization, where an experience becomes a pathology, that is exemplified by the high rates of C-sections and inductions in the U.S., and the second side is a fear of medicalization that affects the decisions clients make regarding their own health. Multiple midwives in this research pointed out this trend, which can be dangerous for a patient when they turn away from providers' advice about necessary medical care because of their fear of medicalization. Midwives must navigate this medicalized birth in healthcare, as well as addressing the concerns of women who may be so worried about the medicalization of their birth that they do not take the advice of their practitioner.

\section{The Medicalized Birth}

Medicalization manifests itself in multiple ways during the birth process, which includes high amounts of inductions, cesarean sections, and the reliance on technology throughout childbirth. In necessary situations these medical advancements are largely beneficial for women, but midwives are advocating for a noninterventionist birth for a mother with no health complications since using these interventions 
has the potential to create problematic issues and increase unnecessary procedures. In addition, insurance in this country has contributed to the medical industrial complex with third-party providers deciding how to price certain procedures, which could bring in more money for hospitals. In turn, this can affect what individuals decide as medically necessary in certain situations, due to falsely connecting the high price with better care, and can lead to more interventions, because they bring in more money for the hospital (Conrad and Leiter 2004). In this culture of medicalization, both doctors and patients routinize low-risk individuals giving birth in hospitals and the accepted use of interventions for women.

When I asked an open-ended question about what medicalization brings to mind, one midwife explains that it "made [her] think the medicalization of birth took away all power. Because it's like it's, they have no power, it's your... You're just like a patient who we have to help because you have this chronic disease, for nine months, you know what I mean" (Midwife Interview 3). This quotation exemplifies how medicalization is perceived by midwives working in the medical field today. The medicalized birth views the patient as having an illness that removes the mother from the process and implements the physician as the decision maker by bringing the birth under the purview of the medical establishment. This subtly tells women their feelings and bodily experiences are not normal and that they need to look to the doctor for decisions rather than advocating for their own autonomy.

One key example of medicalization is the use of inductions, which are a pharmaceutical start to birth where a woman is given different hormones to start contractions. Inductions can be used for medical and non-medical reasons. Midwives pride themselves on striving to give their patients the birth that they want because they put the mother at the head of the birth team; however, many women ask for inductions of labor when it may not be necessary. In the United States in 2018, 27.1\% of births were induced (Martin et al. 2019). Induction increases the chances that an individual will end up having a C-section, which further aligns the birth experience with the biomedical model's dependence on technological intervention (Ruhl and Bingham 2014). Midwives tend to have lower rates of labor induction and embrace a spontaneous start to labor when they can (Attanasio and Kozhimannil 2018). Patients can also ask to be induced for a non-medical reason, which points to people utilizing the technology that is a part of the biomedical model to control their birth. One midwife encapsulates this issue by describing how her patients have increasingly been asking for inductions:

It's been a joke right now running [among midwives] that nobody wants spontaneous labor anymore. Everybody wants to induction between 39 and 40 weeks. And, I mean, not all of our patients, but enough that we're just like, "we have another induction?" I recently had to schedule an induction and our doctors sign off like if they agree or not if they're not... if [the patient is] past 41 days then we can just schedule it. With a 39-week induction for a first-time mom, closed cervix, no real reason, she wanted it because she had an ultrasound and they told her the baby looked big so she... The whole thing was silly, [I tried] to talk her out of it. (Midwife Interview 3)

This quotation illustrates how midwives are stuck in a cycle of wanting to give their patients the type of birth that they would like while at the same time having to contend with medicalization and women asking for these interventions when they are unnecessary. There are multiple reasons why a person could be asking for an induction. One of the main motivations is that individuals want to know when their labor will start because this can ease some anxiety of not knowing. Rather than allowing for spontaneous labor, patients want the certainty of knowing when their baby will arrive. The fact that many times the woman is asking for the induction points to Michel Foucault's theory of biopower (Comaroff and Comaroff 2014). Foucault approaches biopower as a form of power that controls the bodies of the population and is adopted by the individual who then self-corrects and disciplines their own actions after medicalization has taken hold in society (Nadesan 2008). This theory gives a reason for why the women and birthing 
population themselves perpetuate the medicalization that is already inherent within the system. Individuals are correcting their actions to move away from a natural birth. Women are deciding they want inductions themselves, and this puts them into the hospital to have their child under the purview of the medical establishment.

Many of the midwives in this research reference the ARRIVE study, which looked at whether the outcomes for mothers change if a woman is induced or experiences spontaneous labor. The study found that there is not a huge difference in the outcome of the pregnancy if a mother is induced after 39 weeks or not (Carmichael and Snowden 2019). This study has caused providers to think that inductions are fine to use once a woman has reached full term and would not affect the outcome of the birth. Many midwives were unsure of this study because they are hesitant to move away from a non-interventionist stance on normal birth. This is one of many nuanced examples of the medicalization of birth that continues in the healthcare system because inductions that are not needed for a medical reason contribute to the high number of cesarean sections in the United States.

The high cesarean section rate in the United States is another example of how the medicalization of birth is still prevalent in the healthcare system. With the U.S.'s cesarean section rate being so high, this surgical procedure has become something that midwives have to contend with in their working lives as the numbers rise. In times where there is a danger to the mother or the child, this surgery has saved lives, but the availability of the procedure to providers allows for many of them to move toward it before it becomes necessary, exposing mothers to the heightened risks and unintended consequences that can come with a surgical procedure. Midwives who have been working for many years have seen these numbers increase as they have continued in this field. One midwife explains how she has seen the rise in cesarean section rates firsthand due to medicalization:

I've seen an increase in the C-section rate. That's really the major change. I took 14 years off. I worked for a couple years and I had my two kids... and sort of got back on the horse and got privileges in the local hospital as well as in the birth center, and I just see ... so many more inductions, so many more problems and that bothers me because those problems...Some of them are seen by our increase in knowing more about the body, but some of them are...We're not as healthy maybe as we were 20 years ago, right, or less athletic, or you eat more, and those make more problematic clients. But we also have an increased C-section rate and I think that goes hand in hand with epidurals, and the lack of the ability to treat a woman like she's normal, as opposed to like she's a problem. And I think that's really gotten us in the last 20 years. (Midwife Interview 7)

In the face of continued medicalization, midwives are trying to push back against this cultural phenomenon by using their midwifery model. Birth has been brought into the purview of medical management through interventions such as inductions and cesarean sections, but midwives are in a key position to resist these issues. The technology available that makes inductions and cesarean sections possible plays an overall hand in the perpetuation of medicalization in the U.S. healthcare system.

\section{Technology's Role in Medicalization}

The use of technology, such as fetal heart monitors, is intertwined with medicalization and performs a major role in continuing the phenomenon in the United States. The medicalization of birth results in physicians and patients depending on technology for control over the birthing experience, while midwives must work through and avoid the usage of unnecessary technologies that encourage interventions during birth. Midwives can be seen in a negative light for not using these because of the connotation that more technological interventions allow providers and patients to feel a sense of control over the birth, which is an event that is not always predictable. Beginning with the introduction of forceps in the sixteenth century, doctors and surgeons stressed to women that technology that makes birth less of an unknown was accessible only in hospitals, bringing the process of labor into the biomedical model (Sheikh, Ganesaratnam, and Jan 2013). David-Floyd (1994) discusses this 
issue by terming it a technocratic birth, which functions as an agent of social control over individuals, by shaping women's actions and decisions during the birthing experience. Midwives find this to be the case when their model of care argues that these technologies are not always needed.

The relationship between technology and medicalization is fostered through the control these tools can give to providers. One midwife describes this in the following way:

There's also a false sense of security in technology that I feel like, you know, a lot of women go to the hospital because they're being watched, and they're being monitored, and they're being assessed all the time. Because they have the thing on them, and they've got the blood pressure cuff on them, and their IV is going all the time so they're being taken care of. But if you go to the wards you see that nobody's looking at those monitors. They come through and they, you know, look at [a] strip of 20 minutes or the last hour or two and say, "Okay, this looks good." (Midwife Interview 5)

The usage of the term "false sense of security" is very revealing in what midwives feel the downside of the technology can be. Additionally, false positives from these machines can set in motion a host of other technological interventions because people think something is wrong (Bloom, Belfort, and Saade 2016). The invention of these many different tools is a huge leap forward for modern medicine, but they run the risk of detaching providers from the normal process of birth that has been pathologized through medicalization.

Fetal monitoring is a key example of a technology that is a positive tool that can have negative consequences. This tool continues the medicalization process by giving providers a sense of control over the birth process. Birth can be very unpredictable, so tools such as fetal monitoring, which is used to watch the baby's heart rate during labor, can give people a better feeling of what the baby is doing. This tool is considered a standard of care for birth, but constant monitoring can lead to false positives (Bloom, Belfort, and Saade 2016). Midwives tend to use intermittent fetal monitoring, where they look at the baby for twenty minutes and take the machine off for the rest of the hour. Patients see the monitoring as control as well, because birth can be a scary experience. Another midwife explains that:

it also gives the patient... this sense of like everything's okay so it gives them control over it because they can watch the... We literally sometimes have to turn to the screen off, and people are staring at it they don't even know what they're looking at. They don't know how to read it, they don't know if it's good or bad, but it gives people a sense of control. (Midwife Interview 5)

Despite most women not knowing how to read what is coming out of the fetal heart monitor, seeing the lines gives them a belief they have knowledge that things are progressing normally and this feeling of control over their labor. Labor can be an unpredictable event: the culture of fear surrounding it and the history of providers cautioning women against a noninterventionist birth out of the hospital has led to women depending on these machines to feel safe, because they have a sense of control, as the midwife quoted above indicated. Midwives have to work in situations where women and their fellow providers rely on these machines. A significant part of midwives' work is to find a balance between the safe use of these technologies during birth and the slippery slope of unnecessary interventions.

Technology creates this false sense of security because it allows for providers to measure and test throughout the birth. Because women in the United States are having fewer children, the monetary investment into birth is deemed as necessary and the accessibility of obstetric technologies is seen as an assurance of a healthy birth (De Vries 1985). Midwives do not depend on all of the new tools that obstetrics holds in high esteem. This decision can have negative consequences for the midwifery profession because of their belief that birth has the ability to progress without technological assistance (De Vries 1985). Many women want these tools because they want to feel more in control over their labor and birth, which is why they choose the hospital with a physician as a place to give birth over 
midwifery. While today some midwives work in hospitals, consumers continue to overwhelmingly put their money into obstetrics in hospitals, which could take away income for midwives. In hospitals there is a veneration of technology, which comes into contrast with midwifery non-interventionism and is perpetuated through the ideals of safety associated with technology's use and consumer ideology (Wendland 2007). Consumers want to pay the most money to receive the best care, and while these may not always equate, people continue to spend on obstetrics and not on midwifery. Midwives I interviewed suggested that physicians' dependence on technology, a feature of the biomedical model, could stem in part from constant testing during the birth process.

Technology can remove a woman even further from listening to her body, bringing individuals further under the purview of the medical establishment by giving providers more power. By depending on technological interventions, women look to the doctor to interpret the medical outcomes from these machines and fear trusting their body's sensations. Women in labor are excluded as knowledgeable about their own bodies in situations where they have been excluded from the labor process (R. Davis-Floyd and Sargent 1997). A midwife in this research describes the sensations and stress that the technocratic birth process can make individuals feel when creating a barrier between a woman and the non-interventionist model:

I mean, yeah, it's not, it's not useful, it's not necessary, it creates a sense of I mean... I feel like it...it removes the woman from the natural process of birth, because now you've got IV and blood pressure and you know some the pulse [oxygen] and you can't move because when you move then the baby goes off the monitor and people come in because they see that the baby's off the monitor and we got to do something about that, you know, and there's just, I mean I can't imagine having a sensation that you have to use all your strength [to] pay attention to...And having something squeezing my belly constantly, and then moving, and then people coming in and readjusting it while I'm... I mean if that happened to me now I'd be like, "God, geez!", you know, and of course women can't handle it, of course they're going to get an epidural to remove themselves another step. And then the epidural creates the whole cascade of interventions. (Midwife Interview 5)

These technological interventions are features of the modern obstetric environment and contribute to the technocratic birth experience because they remove the patient further from their body and give control to those who can use these tools (R. Davis-Floyd and Sargent 1997). The cutting-edge technology championed in the biomedical model can remove the patient further from the experience of her birth, handing over the decision making to the doctor who can interpret the machineries. These technologies have the potential to keep women safe and bring about positive outcomes if they are used as tools when necessary and not just because they are available and accessible.

Technology has facilitated the medicalization of birth through the development of tools that only doctors in hospitals can use; consequently, women are reassured that the safest birth is in a place where these machines are available. Physicians and patients depend on technology to give them control over the spontaneous thing that is labor and childbirth, while midwives, who want to keep to a non-invasive model of birth, can receive criticism because they do not want to use these technologies all the time. However, as individuals become more aware of the problems that the unnecessary use of technologies can cause, individuals can endanger themselves by ignoring medical advice in an attempt to avoid this.

\section{The Fear of Medicalization}

The medicalization of birth can be dangerous for patients and compromising to midwives when it results in patients rejecting the use of necessary technologies and providers' advice. The medicalization of birth has created a fear among some women that certain interventions will lead to more interference, eventually ending in a C-section. This fear leads to patients being less likely to trust and accept recommendations from their provider, resulting in potentially dangerous situations. Women are trying to take back the decision making and 
leadership role in their birth. Margaret MacDonald (2011), an anthropologist, explains this trend when she states, "the logics of caring and choice emerge as key determinants of the naturalness of birth within midwifery. This is best illustrated when medical technology, nature's erstwhile foe, enters the conversation or, quite literally, the labor room" (4). This overarching anxiety of medicalization stemming from the loss of control has led to problems of patients having their babies outside of the hospital when they need the extra services it has and a higher likelihood of patients refusing transportation to a hospital from a home birth scenario.

When triaging an individual to see if they are right for midwifery care and will not "risk out," midwives run into problems with women who need more expert care but are scared of what it will bring to their birth experience. The term risking out, for example when an individual "risks out" of a midwife's care, means that the individual has certain preexisting conditions that raise the risk of problems occurring during their birth, which is called a "high-risk" birth, and they have a higher chance of needing the technological tools and the specific skill set of a doctor (National Institutes of Health 2017). These can include complications such as a patient pregnant with twins, individuals with diabetes, obesity, preeclampsia, and so on. These clients need more medical expertise because there is a higher chance of a need for an intervention if an emergency arises. Midwives have no control over women who risk out of their care or need to have hospital births after they advise them of this. These individuals may not heed the midwives' advice and seek out alternative care that could put the mother and baby in a dangerous situation.

Midwives see this issue directly because many women who fear medicalization reach out for their services first with the goal of having a less interventionist birthing experience. Some women have seen the 2008 Ricki Lake documentary The Business of Being Born, which details how the United States healthcare system views childbirth as a moneymaking opportunity set on a schedule, and this has affected their decisions when looking at their own birth. The midwives in this research have not only pointed out the problems with how the United States approaches birth that this documentary circulates to a wider audience, but also they note the difficulties that it has created in their relationships with patients. One midwife describes these obstacles well:

When we get a like mid-pregnancy transfer for non-logistical reasons like not somebody who has moved or something, but somebody who just wants to change care provider, the number one reason they give for why they're coming to us is that they saw The Business of Being Born and are like now scared by their provider and want an out of hospital birth or, you know, midwifery care... so it's a cool movie, and they make some really good points ... it's not out of nowhere but it's scared a lot of people into thinking that one intervention is going to yield the next and it's, it's difficult because on the one hand, there are people who definitely do it that way. And on the other hand, sometimes the intervention cycle, the spiral starts because like things aren't going well. (Midwife Interview 2)

Midwives want to be available for women who do not have preexisting medical conditions that would need a more hands-on birth from a physician, but some individuals may still require a physician and hospital services if complications arise. This situation puts midwives in a hard spot because if a patient needs a higher level of care but fear a different provider will lead to them having a medicalized birth, they might seek other services that may not be accredited or safe. This situation drives midwives to create techniques to build a relationship with their patients so their advice will be heeded.

The midwives I spoke with discussed different techniques they use to prepare and build trust with their patients to ease their worries and ensure that patients will listen to the midwives' recommendations. Midwives want to provide the best level of care and building trust with clients is important because midwives need their patients to respect and heed their professional advice. A midwife in this research describes a scenario where she 
received some pushback from the partner of her client:

She had not been progressing for a long time and then we recommended an epidural. Before we did the epidural, we did some things to help the positioning, you know, and then another four hours passed without her dilating at all so I was like "uhhh, it's time for some Pitocin," and her partner was like, "I don't know why we got to go straight to the drugs," and I was like "ooooooh, we did NOT go straight to the drugs, sir." (Midwife Interview 2)

This midwife received a hostile response from her patient's partner who wanted to advocate for her patient, and she had to navigate a situation where she faced an uphill battle in giving this woman interventions that she needed for her health. This fear of medicalization has affected the relationships between the provider and patient, making the job of the provider more complicated. Another midwife describes what worries her when people who need that extra care do not heed their provider's advice:

We've had cases where we have told patients that they are not good candidates for midwifery care or birth center because they have like risked out. And you need care beyond either what [midwives] can provide or what we can provide in our [birth] center and every now and then we have one of those who goes and finds a certified professional midwife to deliver with which is just like really scary. (Midwife Interview 2)

Women who turn to certified professional midwives when they require the care that only hospitals and physicians can provide can create a negative connotation for the rest of the midwifery profession. When a client chooses to go against medical advice, and then a midwife decides to take them on, it could affect the rest of the profession due to the potential for the birth to end in disaster. People then point to all of midwifery as the culprit for the danger and this negative connotation continues to affect the way midwives are accepted into society. This issue could perpetuate the biomedical model being viewed as superior in the United States' culture because it creates the perception that different styles of birth are excessively risky (Lee et al. 2019). The midwifery model of care puts women as the decider in their birth experience, which could be interpreted by other providers as unnecessarily risky.

This fear of medicalization can result in individuals refusing routine tests that check their health and that of their baby. Midwives still use tests to check the progression of the pregnancy and make sure the mother does not have any underlying conditions that could require a physician's care. A midwife in this study describes how she wants to be able to give her clients the experience they came to her for, but this can be complicated:

There are things they don't want you know... perfect example is always the glucose test; nobody ever wants to do it. [Lots of our patients have] read online somewhere, that is horrible for you and it has ... I don't know what in it, you know, really it's sugar it's very sugary water. We have the kind that's like no additives, no preservatives like really like doesn't even have dye in it, clear. And people don't want to take it. And it's really frustrating because sometimes they walk in drinking like a soda or juice and you're like, it's the same thing. But when we talk about that one... I'm like, that's the only test and we have the evidence-base to be able to diagnose diabetes. (Midwife Interview 3)

This anxiety about having a medicalized experience can make women feel that different tests will lead them down a path of a fully medicalized birth. In some cases, the tests that midwives use may reveal that patients require a physician's care during childbirth, but these tests are necessary for a certain standard of care in pregnancy. The fact that the biomedical model depends on the analysis of different tests can continue this fear that women have toward the medicalized birth, causing them to resist the necessary level of adequate care.

Apart from testing, the discussion of transportation to a hospital from a home birth or birth center setting is a typical moment where women worry they will end up having a medicalized birth experience. While some of these transportations are responses to emergencies, midwives often suggest a 
preventive move to the hospital in order to prepare for a potential emergency, bringing up possible situations to prepare the mother. The goal is to have the mother ultimately make the call based on her midwives' advice, but due to this fear of medicalization that happens in hospitals, patients may decide against going when it is a necessary next step. One midwife discusses a hard situation she was put in because of a client that was concerned about abandoning her home birth plan:

So one of our contracts that [patients] sign with us is that, that if we think it's necessary to transfer we'll say that and we want them to support us in that but just because they signed my contract at the beginning doesn't mean we're going to necessarily follow through. And I never had to leave a client, but it crosses my mind, sometimes, and it would be desertion. Right. So, I had a situation in the summer where maybe the baby had had some questionable heart tones, and we had done a little monitoring and it was all fine and good, but I said "I'm not sure this is [the best home birth situation]..." (client replies), "No, no, we're staying home, we're staying home"... I talked with all my peers, like, what do I do if, if, I think it's time to go, and she doesn't. Where does it...Where is it desertion versus I can't care for you anymore because this is not healthy, you know, and I think my boss said, "Call an ambulance and tell the ambulance that she's theirs. And then leave." (Midwife Interview 7)

In all of these patient relationship scenarios, midwives are put into complicated situations because of their patients' fear of medicalization. These women worry that once they arrive at a hospital, they will be buried under interventions that will eventually lead to a C-section. While midwives want women to be in charge of their own experience, this anxiety surrounding medicalization can impact the ability of providers to do their job. MacDonald explains a solution to this issue when she discusses how "[a woman] makes sense of her request [for intervention] as an 'informed choice,' carefully distinguishing her experience from the interventionist routine she had previously experienced" (MacDonald 2011, 5). Women want to feel control over their birthing experience, which is why making these decisions as an informed choice that a woman can make with advice from her midwife could address this fear. This means including women in the entire birth process and making the experience more of a joint effort between the provider and the mother, rather than delegating everything to the professional. The medicalization of birth will continue to affect the relationships between provider and patient, and patients' fear of this process can cause multiple problems for providers who aim to give their patients the best care possible.

\section{Conclusion}

The medicalized birth in the U.S. has been propelled by advancements in obstetrical technologies that intend to make birth less dangerous and unpredictable, but midwives have seen a negative side to this; some women fear interventions so much so that they ignore necessary medical advice. Both the typical embodiment of medicalization, exemplified by the high rates of $\mathrm{C}$-sections and inductions in the U.S., and the backlash that some patients show to this phenomenon through their fear of medicalization in turn affects their healthcare decisions, is something that midwives see consistently in their work. Michel Foucault's theory of biopower (Comaroff and Comaroff 2014) and Robbie David-Floyd's idea of the technocratic birth (1994) can help explain how or why women are making their decisions, by describing how medicalization interacts with technology and why women fear interventions leading to the hyper-medicalization of. Midwives strive to find a middle ground between using necessary technologies and obstetrical tools to assist women in birth without raising the $\mathrm{C}$-section rate, all while a client has their own needs and wants for their birth experience.

While technology can have a positive impact, practitioners in birth need to be aware and cautious of their reliance on these tools in their work because this can lead to distrust from women who see this as negatively affecting their birthing experience. These issues can also affect problems with access to healthcare in the United States, specifically concerning cost and coverage, which could be discussed in further 
research. Additionally, these technologies affect the culture of birth across the country by enabling the medicalization of birth. This can adversely affect the rates of unnecessary interventions for women, such as C-sections and labor inductions, making the natural experience of birth much more dangerous than it needs to be. 


\section{Acknowledgements}

Thank you to my professors, Dr. Friederic and Dr. Jones, who have guided me through college, made me into the anthropology student that I am, and pushed me to ask more questions about the world around me. Mom, Dad, and Jean thank you for supporting me as I pursue a career that makes me excited for the future. 


\section{References}

American College of Midwives. n.d. "Become a Midwife." American College of Midwives. Accessed April 20, 2020. https:// www.midwife.org/Become-a-Midwife.

Attanasio, Laura, and Katy B. Kozhimannil. 2018. “Relationship Between Hospital-Level Percentage of Midwife-Attended Births and Obstetric Procedure Utilization." Journal of Midwifery \& Women's Health 63 (1): 14-22. https://doi.org/10.1111/ jmwh.12702.

Bloom, Steven L., Michael Belfort, and George Saade. 2016. "What We Have Learned About Intrapartum Fetal Monitoring Trials in the MFMU Network." Seminars in Perinatology 40 (5): 307-17. https:// doi.org/10.1053/j.semperi.2016.03.008.

Brodsky, Phyllis L. 2008. "Where Have All the Midwives Gone?" The Journal of Perinatal Education 17 (4): 48-51. https:// doi.org/10.1624/105812408X324912.

Capitulo, Kathleen. 1998. "The RISE, FALL, and RISE of NurseMidwifery in America | Ovid." The American Journal of Maternal/ Child Nursing 23 (6): 314-21.

Carmichael, Suzan L., and Jonathan M. Snowden. 2019. “The ARRIVE Trial: Interpretation from an Epidemiologic Perspective." Journal of Midwifery \& Women's Health 64 (5): 657-63. https:// doi.org/10.1111/jmwh.12996.

Centers for Disease Control and Prevention. 2019. "Births - Method of Delivery." Accessed November 27, 2019. https://www.cdc.gov/ nchs/fastats/delivery.htm.

Centers for Disease Control and Prevention. 2020. “NVSS - Birth Data." Accessed March 25, 2020. https://www.cdc.gov/nchs/nvss/ births.htm.

Comaroff, Jean, and John Comaroff. 2014. "Introduction of Revelation and Revolution." In Anthropology in Theory: Issues in Epistemology, edited by Henrietta L. Moore and Todd Sanders, 309-19. 2nd ed. Chichester, West Sussex; Malden, MA: Wiley Blackwell.

Connerton, Winifred. 2012. "Midwifery." Encyclopedia Britannica. May 25, 2012. https://www.britannica.com/science/midwifery. 
Conrad, Peter, and Valerie Leiter. 2004. "Medicalization, Markets and Consumers." Journal of Health and Social Behavior 45: 158-76.

Davis-Floyd, Robbie. 1987. "The Technological Model of Birth." The Journal of American Folklore 100 (398): 479-95.

Davis-Floyd, Robbie E. 1994. "The Technocratic Body: American Childbirth as Cultural Expression." Social Science \& Medicine 38 (8): 1125-40. https://doi.org/10.1016/0277-9536(94)90228-3.

Davis-Floyd, Robbie, and Carolyn Fishel Sargent, eds. 1997. Childbirth and Authoritative Knowledge: Cross-Cultural Perspectives. Berkeley: University of California Press.

De Vries, Raymond G. 1985. Regulating Birth: Midwives, Medicine, \& the Law. Health, Society, and Policy. Philadelphia: Temple University Press.

Declercq, Eugene. 2015. “Midwife-Attended Births in the United States, 1990-2012: Results from Revised Birth Certificate Data." Journal of Midwifery \& Women's Health 60 (1): 10-15. https:// doi.org/10.1111/jmwh.12287.

Johanson, Richard, Mary Newburn, and Alison Macfarlane. 2002. "Has the Medicalisation of Childbirth Gone Too Far?" BMJ: British Medical Journal324 (7342): 892-95.

Lee, Suzanne, Des Holden, Rebecca Webb, and Susan Ayers. 2019. "Pregnancy Related Risk Perception in Pregnant Women, Midwives \& Doctors: A Cross-Sectional Survey." BMC Pregnancy and Childbirth 19 (September). https://doi.org/10.1186/s12884019-2467-4.

MacDonald, Margaret. 2011. "The Art of Medicine: The Cultural Evolution of Natural Birth." The Lancet 378 (9789): 394-95.

MacDorman, Marian, and Eugene Declercq. 2019. "Trends and State Variations in Out-of-Hospital Births in the United States, 20042017." Birth (Berkeley, Calif.) 46 (2): 279-88. https:// doi.org/10.1111/birt.12411.

Martin, Joyce, Brady Hamilton, Michelle Osterman, and Anne Driscoll. 2019. "National Vital Statistics Reports for Births: Final Data for 2018." Volume 68, Number 13. Centers for Disease Control and Prevention. https://www.cdc.gov/nchs/data/nvsr/nvsr68/ nvsr68_13-508.pdf. 
Nadesan, Majia Holmer. 2008. Governmentality, Biopower, and Everyday Life. London: Taylor \& Francis Group.

National Institutes of Health. 2017. "What Is a High-Risk Pregnancy?" Last modified January 31, 2017. Accessed February 2, 2021. https://www.nichd.nih.gov/health/topics/pregnancy/ conditioninfo/high-risk.

Parry, Diana C. 2008. "We Wanted a Birth Experience, Not a Medical Experience': Exploring Canadian Women's Use of Midwifery." Health Care for Women Internationa/29 (8-9): 784-806. https:// doi.org/10.1080/07399330802269451.

Ruhl, Catherine, and Debra Bingham. 2014. "Midwives and Nonmedically Indicated Induction of Labor." Journal of Midwifery \& Women's Health 59 (3): 233-36. https://doi.org/10.1111/ jmwh.12197.

Sheikh, Sukhera, Inithan Ganesaratnam, and Haider Jan. 2013. "The Birth of Forceps." JRSM Short Reports 4 (7): 1-4. https:// doi.org/10.1177/2042533313478412.

Wendland, Claire. 2007. "The Vanishing Mother: Cesarean Section and 'Evidence-Based Obstetrics."' Medical Anthropology Quarterly 21 (2): 2018-2233.

WHO. 2015. "WHO Statement on Caesarean Section Rates." World Health Organization. Accessed March 21, 2020. http:// www.who.int/reproductivehealth/publications/ maternal_perinatal_health/cs-statement/en/. 